\title{
A new islanding detection scheme based on combination of slantlet transform and probabilistic neural network for grid-tied photovoltaic system
}

\begin{abstract}
According to find islanding in a grid tied photovoltaic system, a combination of Slantlet Transform (SLT) and Probabilistic Neural Network (PNN) has been proposed in this paper. The SLT is used to extract the unique features from three phase voltage signals at the Point of Common Coupling (PCC). The produced features vector is fed to a probabilistic neural network classifier as input pattern which is used to predict islanding condition. The proposed technique is validated through comparing with islanding response time in different situations with the offered response time through Rate of Change of Frequency (ROCOF) method.
\end{abstract}

Keyword: Detect islanding technique; Photovoltaic systems; SLT; PNN 